\title{
NEUTRON SCATTERING TECHNIQUES APPLIED TO MARTENSITIC TRANSFORMATIONS AND SHAPE MEMORY ALLOYS
}

\author{
W. B ̈̈HRER and M. ZOLLIKER \\ Labor für Neutronenstreuung, ETH Zürich, CH-5232 Villigen PSI, Switzerland
}

\begin{abstract}
The advantages of thermal neutron scattering in materials science are shortly reviewed. Recent results obtained on the shape memory alloys $\mathrm{Cu}-\mathrm{Zn}-\mathrm{Al}$ and $\mathrm{Cu}-\mathrm{Zn}$ are presented and discussed. Emphasis is given to new, 'non-standard' techniques for the determination of effects of stacking faults on peak positions and intensities, short-range order and static displacements through elastic diffuse scattering, and temperature and concentration dependence of phonon dispersions close to the martensitic phase transition.
\end{abstract}

\section{Preface}

The aim of this paper is neither the presentation of the 'art of neutron scattering' nor a review of 'neutrons and shape-memory alloys'; we rather want to show that, among other techniques like $\mathrm{x}$-rays or electron-microscopy,

neutron scattering is a very efficient tool

for the investigation of metallic alloys with martensitic phase transitions.

\section{Introduction}

Experiments with thermal neutrons are most useful in the study of condensed matter. To a first approximation, a neutron impinging on a sample is scattered by the nuclei, and the energy and momentum of the scattered neutron is determined by the static and dynamical behavior of the sample's nuclei. The advantage, as compared to other techniques, stems from the fundamental nature of the neutron: the de Broglie wavelength of a thermal neutron is of the same order of magnitude as the interatomic distances in crystals, and the energy of a thermal neutron is of the same order as the energy of a normal lattice excitation. Therefore in a scattering process, their exist pronounced interference effects which allow the determination of atomic positions and of atomic motions. In the neutron experiment, one actually measures count rates which are determined on one hand by the experimental setup, i.e. incoming flux, angular and energy resolution etc., and on the other hand by the double differential scattering crosssection which describes the sample and the neutron-nucleus interaction. The basic problem is to derive theoretical expressions for this quantity, appropriate to the problem to be solved; relevant cross-sections for the different types of neutron scattering - coherent, incoherent, elastic, inelastic, diffuse, etc - are given in $/ 1,2,3 /$.

Conceptually the scattering process can easily be separated into the individual contributions, the 'practical' use of the formulae however is not a simple task. Shape memory (SM) alloys behave, at least as far as we can judge from the 'neutron scatterer's viewpoint', in a 'non-standard' manner, they are more complicated than the perfect crystal systems, treated in standard crystallography and solid state physics. Therefore for an understanding of an SM 
alloy, it is generally necessary to have information from different types of neutron experiments as well as from other techniques.

The neutron is uncharged, it penetrates deeply into the target and allows the material to be studied without limitations with respect to the surface. Neutrons are unique in observing bulk average properties on the scale of centimeters. This means that interior properties of components can be studied without careful cutting procedures. The low absorption of neutrons in most materials makes sample environment control a much easier and broader field than is possible with competing materials research techniques. 'In situ' experiments within furnaces, cryostats and pressure cells are all routine in the neutron field.

One disadvantage of neutron scattering is the relative low intensity of available neutron beams. This necessitates having a large experimental facility, for example a nuclear reactor or a spallation source, thus making the method somewhat exclusive.

In the following we restrict ourselves to copper-zinc-aluminium alloys. We start the discussion by presenting the results of some 'macroscopic' experiments, followed then by 'microscopic' investigations. In our terminology macroscopic refers to investigations and characterizations of 'real' SM samples; whereas 'microscopic' refers to more basic research like determination of atomic positions and lattice vibrations, which however is crucial for the interpretation of the 'macroscopic' data.

\section{Macroscopic}

\subsection{Hysteresis of martensitic transformation}

The martensitic phase transition is first order and, by cycling through the transition, a hystersis is observed. An additional broadening of the hysteresis might be due to concentration inhomogenities in the alloy $(\mathrm{Cu}-\mathrm{Zn}-\mathrm{Al}$ or $\mathrm{Cu}-\mathrm{Zn}$ with a strong concentration dependence of the $M_{S}$ temperature), furthermore the needle- or lens-like growth of the martensite with many phase boundaries can give additional changes of lineshapes. Fig. 1 shows hysteresis curves, as measured on $\mathrm{Cu}-\mathrm{Zn}-\mathrm{Al}$ samples of approx. $1 \mathrm{~cm}^{3}$ volume. The data are actually taken on single crystals, but the method works as well on powder samples. Plotted are neutron intensities of austenitic Bragg reflections which are a direct measure of the relative volume of that phase (similar runs can be performed on the martensitic reflections, the selection is made through observations at a given scattering angle $2 \Theta$. Hysteresis curves of very different widths (Fig. 1a: $18 \mathrm{~K}, 1 \mathrm{~b}: 35 \mathrm{~K}$ ) and different shapes are observed (e.g. Fig. 1b, very broad wings). In the context of a two-way memory application, it is evident that these findings are important and that questions to the 'why' or 'how to avoid' should be answered.
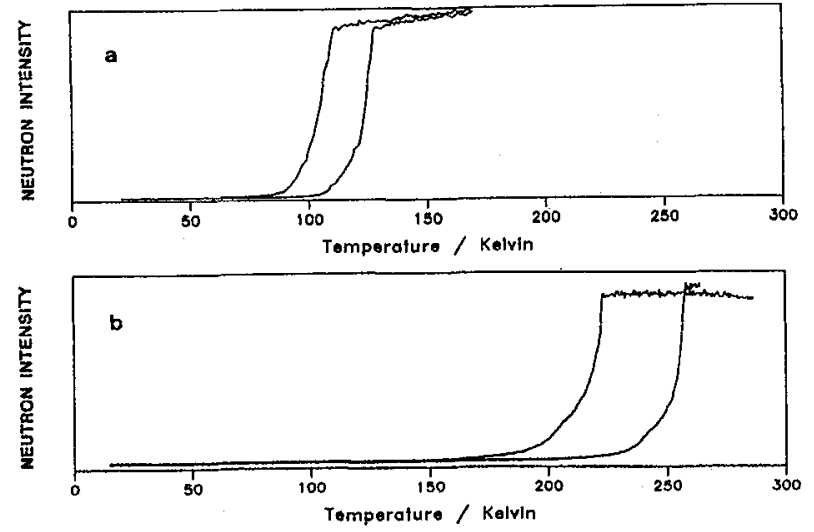

Fig. 1: Neutron intensities, measured on single crystals as a function of temperature; the maximum of the (200) austenite Bragg peak is plotted for two $\mathrm{Cu}-\mathrm{Zn}-\mathrm{Al}$ samples of different composition

a) $\mathrm{Cu}_{.68} \mathrm{Zn}_{.17} \mathrm{Al}_{.12}$, and

b) $\mathrm{Cu}_{.63} \mathrm{Zn} .25 \mathrm{Al} .12$, both with 'unknown history'; note different hysteresis width and broad wings in $b$ ). 


\subsection{Phase indentification $(\alpha, \beta)$}

In the concentration range near to the martensite, the $\beta$-phases of $\mathrm{Cu}-\mathrm{Zn}-\mathrm{Al}$ - and $\mathrm{Cu}-$ $\mathrm{Zn}$-alloys are not stable, they recrystallize into the fcc $\alpha$-modification. On one hand, for basic research one certainly likes to have a pure $\beta$-phase sample; on the other hand however, the mechanical properties of $\alpha$ - and $\beta$ phases are very different, and for the ease of working, a certain amount of $\alpha$ phase is desired. The relative amounts of $\alpha$ - and $\beta$-phase are controlled by the cooling rate, which itself depends on the grain size, on $\mathrm{M}_{\mathrm{S}}$, on the control of atmosphere (loss of $\mathrm{Zn}$ ), etc. Neutron diffraction allows an easy and quick determination; examples are shown in Fig. 2a and b. Intensities are plotted as a function of the scattering angle; sample sizes are about $2 \mathrm{~cm}^{3}$, counting times about $1 \mathrm{~h}$ (with the multidetector instrument at the reactor Saphir, Würenlingen). By calculating the structure factors and comparing the integrated intensities, the $\alpha$-phase concentrations can easily be determined: they amount to $55 \%$ for Fig. $2 a$, and are $<1 \%$ for Fig. $2 b$. The very good sample $b$ was used for the subsequent structure determination of the martensite (Sect. 3.1)
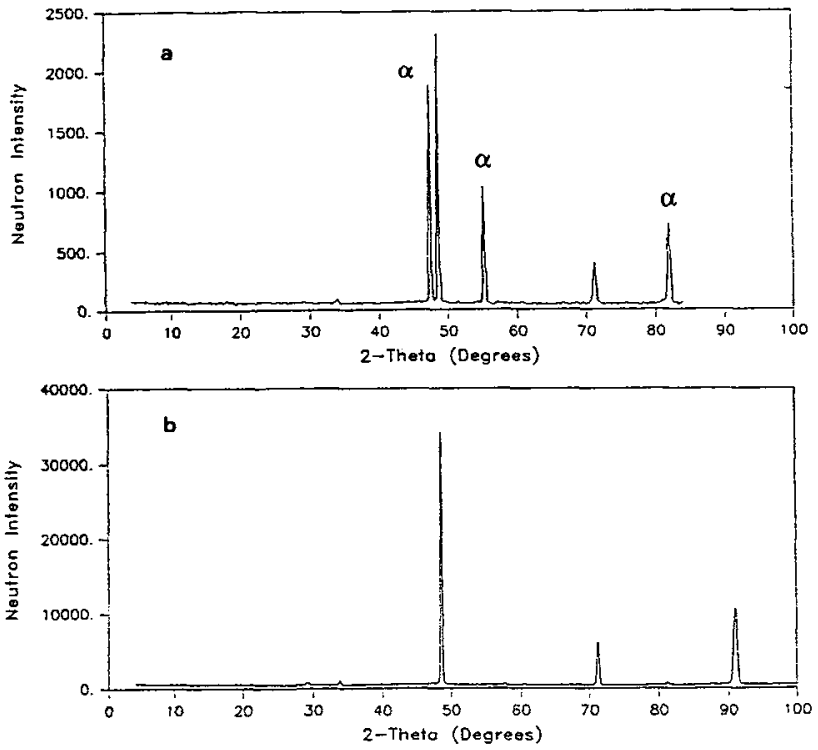

Fig. 2: Neutron intensities, plotted as a function of scattering angle $2 \theta$, for two $\mathrm{Cu}-\mathrm{Zn}-\mathrm{Al}$ powders after different heat treatments; note the concentration of the $\alpha$-phase:

a) $\approx 55 \%$, b) $<1 \%$.

\subsection{Preferred orientation, domains (effect of training?)}

Mechanical parts, to be used in shape memory applications, have to be 'trained' in order to get best efficiencies. We investigated 2 polycrystalline samples of $\mathrm{Cu}-\mathrm{Zn}-\mathrm{Al}$, approx. $3 \times 30 \times 0.8 \mathrm{~mm}^{3}$, one in the 'as recieved' state, the other after a thermomechanical treatment. The measured Debye-Scherrer diagrams are shown in Figs. 3a to e, where the intensities for the austenitic and the martensitic phase of both samples are plotted. The comparison of the figures shows that the Bragg peaks agree with respect to the angular positions, but that the intensities are very different. According to the theoretical cross-section, integrated peak intensities are basically determined by the atomic positions in the unit cell; however the comparison of sample 2 in the 'vertical' and the 'horizontal' set-up, Fig. 3d and e, shows that in addition to atomic positions other effects such as preferred orientation, type of defect and defect concentration etc. are very important. 

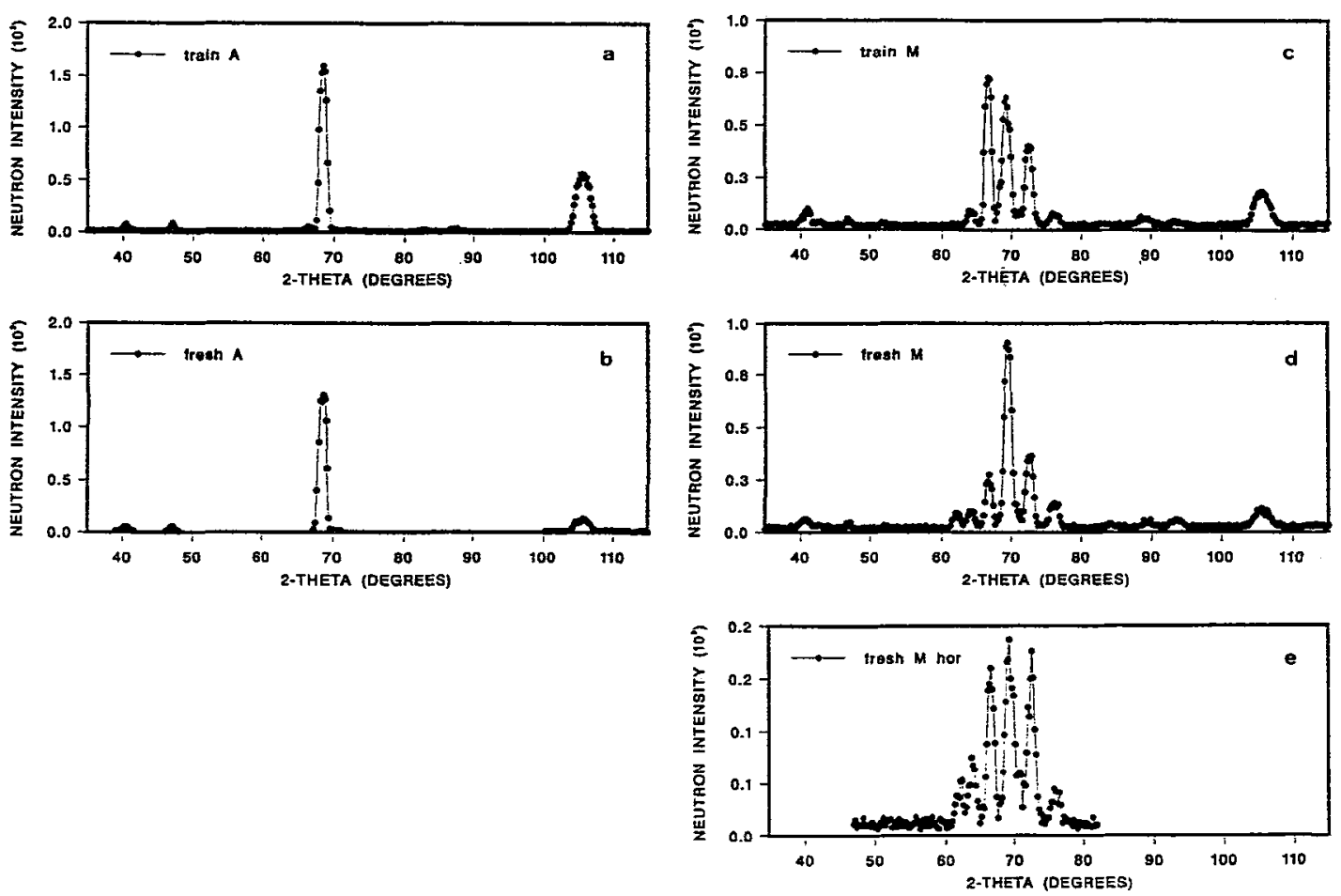

Fig. 3: Neutron intensities, plotted as a function of scattering angle $2 \theta$; polycrystalline $\mathrm{Cu}-\mathrm{Zn}-\mathrm{Al}$ strips, $M_{S} \approx 355 \mathrm{~K}$; experiments at $295 \mathrm{~K}$ and $385 \mathrm{~K}$; a) 'trained', austenite, vertical ; b) 'fresh', austenite, vertical; c) 'trained', martensite, vertical; d) 'fresh', martensite, vertical; e) 'fresh', martensite, horizontal; the terms vertical and horizontal refer to the orientation of the long axis of the strips; note agreement in peak positions, but strong disagreement in peak intensities within the two phases.

In order to understand these diagrams, we need a 'reference' system, which allows us to classify the qualitative differences; in other words, we have to start a 'microscopic' investigation of a 'perfect' $\mathrm{Cu}-\mathrm{Zn}-\mathrm{Al}$ powder sample.

\subsection{Discussion}

The few examples discussed above show that neutron scattering can give interesting and new information on SM samples. For an understanding on a qualitative basis, a series of experiments on well defined materials (concentration, thermo-mechanical 'history', etc.) is already informative. For the interpretation on a quantitative basis, much more work has to be done: effects of strain, defects (type and concentration), grain size, etc. have to be investigated and cast into analytical expressions in order to interprete the experimental data. A first attempt, the influence of stacking faults on powder diagrams, is treated in some detail in Sect. 3.1. 


\section{Microscopic}

\subsection{Crystal structure (average, long range order)}

A carefully prepared powder sample of $\mathrm{Cu}-\mathrm{Zn}-\mathrm{Al}$ has been investigated by elastic neutron diffraction. The Debye-Scherrer diagram of the austenitic phase is shown in Fig. 4a. The analysis with site occupancies gave the following result: $\mathrm{DO}_{3}$ structure with maximal degree of order, $\mathrm{Cu}$ on sublattices 3 and 4 (full occupancy), the remaining $\mathrm{Cu}$ and part of $\mathrm{Zn}$ on sublattice 2, and $\mathrm{Zn}$ and $\mathrm{Al}$ (exclusively) on sublattice 1. The martensite diagram is plotted in Fig. 4 b. The analysis of this diagram could not be performed with the standard powder programs used in crystallography. First the peak shape had to be approximated by a Voigtcurve (convolution of a Gaussian and a Lorentzian), this unusual behavior might be due to size effects of the grains and diffuse scattering contributions, resulting in an ill defined background. But even with improved peak shapes some dicrepancies in peak intensities and positions remained between experiment and calculation remained. We had to introduce stacking faults in order to account for these effects. Fig. $4 \mathrm{~b}$ shows the result of the best fit, where a satisfactory agreement is obtained (plot at the bottom). The most probable stacking fault is of the type $21(31)$ as shown on the right of Fig. 4b, with a concentration of about $4 \% / 4 \%$.
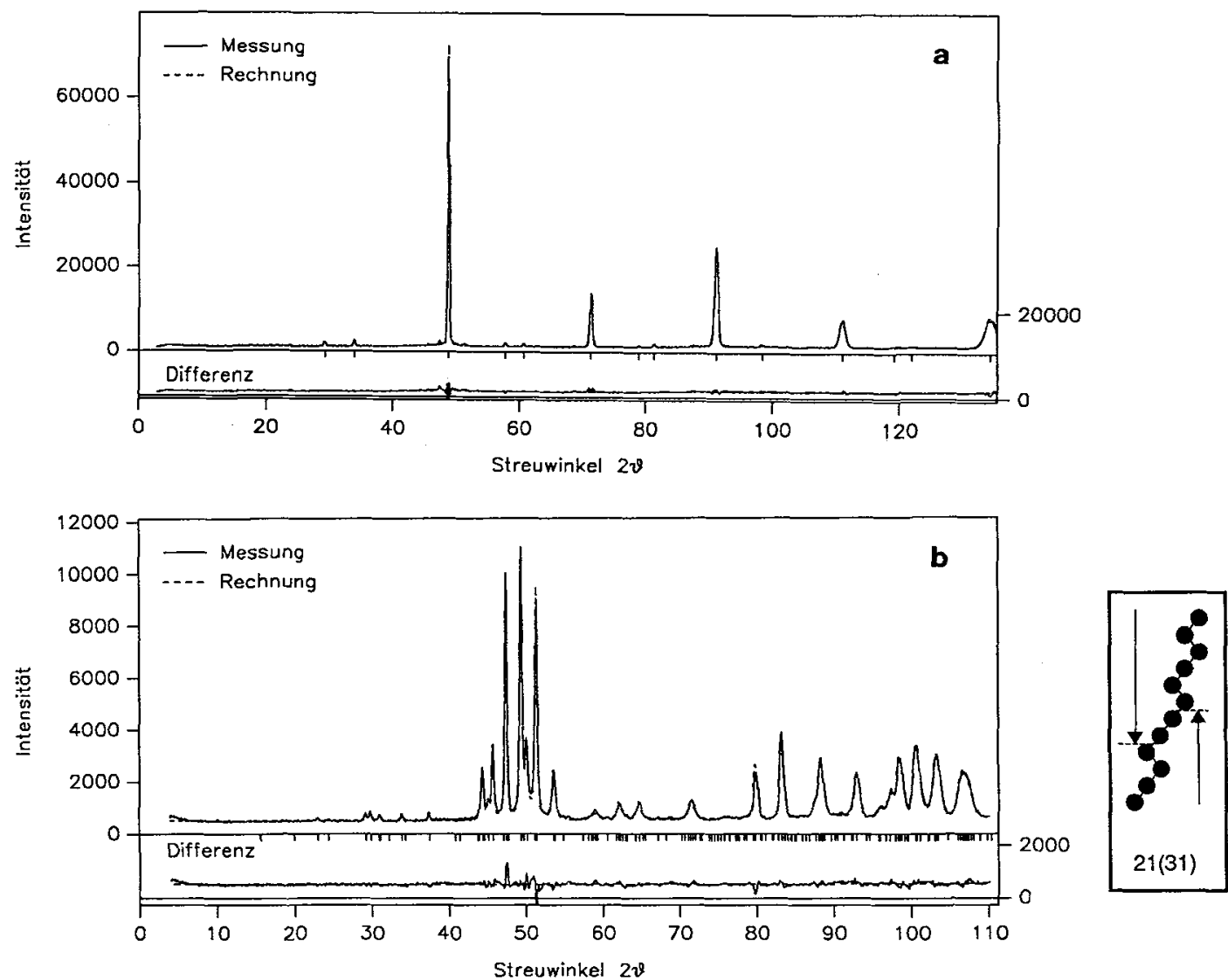

Fig. 4: Neutron diffraction diagramms for a $\mathrm{Cu}-\mathrm{Zn}-\mathrm{Al}$ powder sample, $\lambda=1.709 \AA$; comparison with calculations; a) austenite $395 \mathrm{~K}$; b) martensite $10 \mathrm{~K}$, model with about $4 \%$ stacking faults of the type shown on the right. 


\subsection{Local Structure (short range order)}

A non-stoichiometric alloy cannot have a perfect local structure: mismatch of atomic sizes and/or short range order effects will create small distortions with respect to the long range average structure, as determined by the diffraction experiments. In an elastic neutron scattering experiment the 'average' crystal structure gives the Bragg peak intensities, but the deviation from the average gives additional intensity between the Bragg peaks (in reciprocal space). If some correlations with respect to order and displacements exist, this intensity will be modulated. Experiments to measure these contributions have been performed for $\mathrm{Cu}-\mathrm{Zn}-\mathrm{Al}$ and $\mathrm{Cu}-\mathrm{Zn}$ on single crystals in the austenitic phase. In order to determine the 'real' coherent elastic diffuse components of the intensity (i.e. that due to ordering effects) and to avoid any inelastic contributions by phonons, the experiments were performed on a triple axis spectrometer with energy analysis. The spectrometer was equipped with a Eulerian cradle in order to measure the full angular range. The data were calibrated by measuring the incoherent scattering from a vanadium cylinder of the same outer dimensions and same alignment as the sample. The data were corrected for background, absorption and multiple scattering as well as for incoherent contributions.

The elastic diffuse scattering is shown in Fig. $5 \mathrm{a}$ for the (001) planes of $\mathrm{Cu}-\mathrm{Zn}-\mathrm{Al}$, and Fig. $5 \mathrm{~b}$ for $\mathrm{Cu}-\mathrm{Zn}$. Overall, the same features are observed: a ridge between the (200) and the (110) Bragg reflections, caused by displacement scattering (symmetry considerations) and low scattering intensity elsewhere, indicating a high degree of long-range order. Regions marked hatched have very strong Bragg contributions and have been omitted in the analysis.

$\mathrm{The} \mathrm{Cu}-\mathrm{Zn}$ data were treated in more detail. The intensities were analysed considering short-range order and an expansion of the static displacements up to the quadratic terms $15 \%$. The Fourier coefficients of the resulting 10 Fourier series were obtained by least-squares fitting. The first short-range order parameter has a value of about 0.4 which is consistent with a high degree of long-range order; the low value of the parameters for the neighbouring shells indicates a nearly statistical uncorrelated distribution of the surplus $\mathrm{Cu}$ atoms on the sites of the
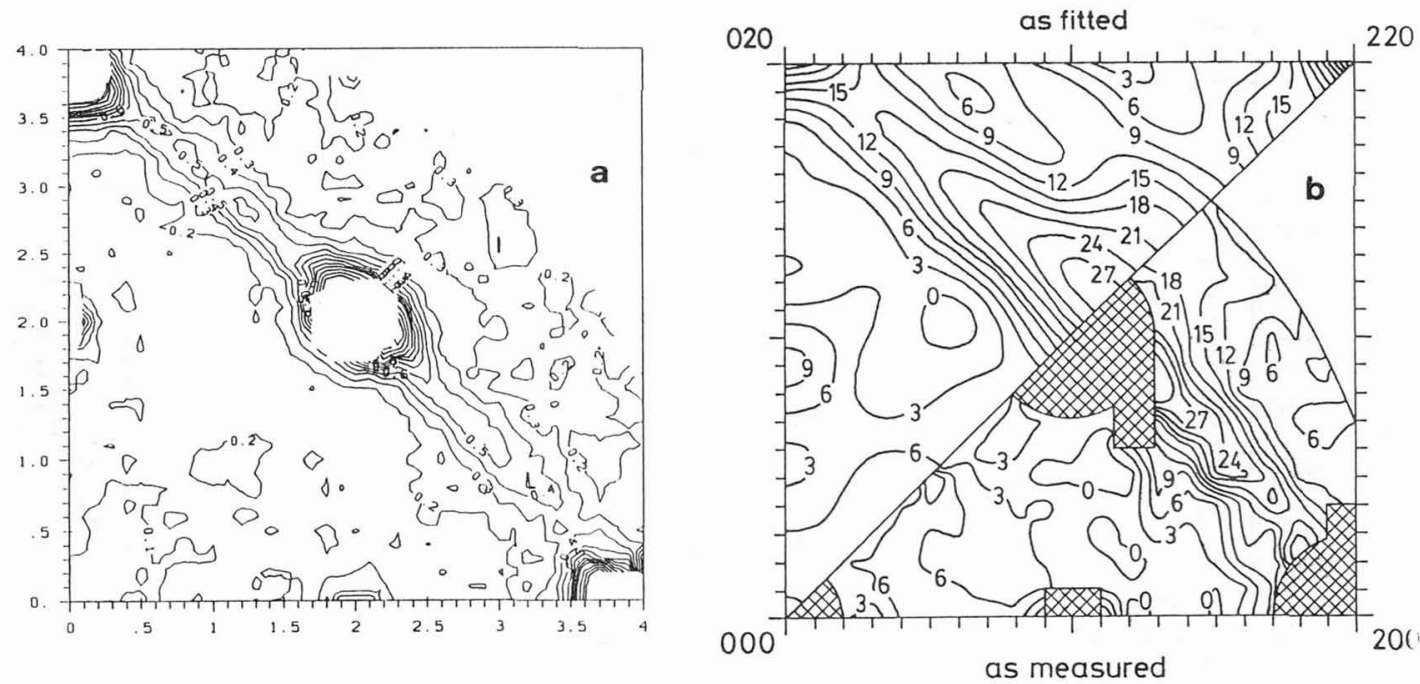

Fig. 5: Elastic diffuse intensity of austenitic $\beta$-phases in the $(100)$ plane of reciprocal space (in Laue units); Bragg peaks omitted (hatched regions); a) $\mathrm{Cu}-\mathrm{Zn}-\mathrm{Al}$; b) $\mathrm{Cu}-\mathrm{Zn}$ : comparison of experiment (lower triangle) and calculation (upper triangle), based on short-range order and displacement contributions. 
$\mathrm{Zn}$ atoms in the B2 structure. Although the measurements were done close to the direct beam, quadratic displacement terms had to be included to improve distinctly the quality of the fit. This indicates a high correlation of the atomic displacements. The term for linear displacement scattering is dominant and indicates rather high values for the mean atomic displacements.

\subsection{Lattice instability, Phonon dispersion}

Precursor effects of the low temperature phase, such as anomalies in elastic constants or phonon dispersion have been observed in different compounds with varying strength. However their role in first-order displacive phase transitions remains controversial.

A simple member of the SM-family is copper-zinc with a composition near 40 at.\% $\mathrm{Zn}$. The $\{110\}$ planes of the austenite correspond to the habit planes of the nearly close packed structure of the martensite.

The phonon dispersion curves were measured by inelastic neutron scattering along the directions [100] and [110]. As a 'reference system', we use the stoichiometric alloy $\beta \mathrm{CuZn} \mathrm{/6/}$ which does not show a low temperature phase transition. In Fig. 6, the dispersion curves along the direction [100] are plotted. The frequencies of the 'shape-memory'- and the 'normal' alloy agree within the experimental error suggesting that mass change and disorder have only a small effect on the average crystal. A characteristic feature of bcc metals is the low frequency of the transverse mode $\mathrm{TA}_{2}$ along direction [110] (polarized parallel to [110]). Fig. 7 shows the comparison of the two alloys: the SM-alloy frequencies are exceptionally low, about $15 \%$

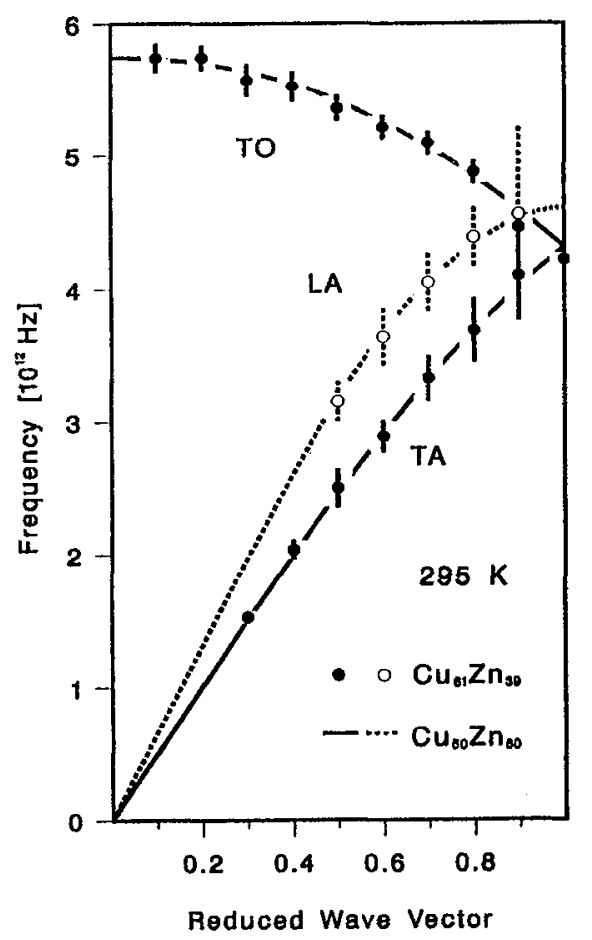

Fig. 6: Phonon branches TA, LA and TO along the [100] direction of the SM alloy $\mathrm{Cu}-\mathrm{Zn}$ at $295 \mathrm{~K}$ compared with stoichiometric $\mathrm{CuZn}$. 


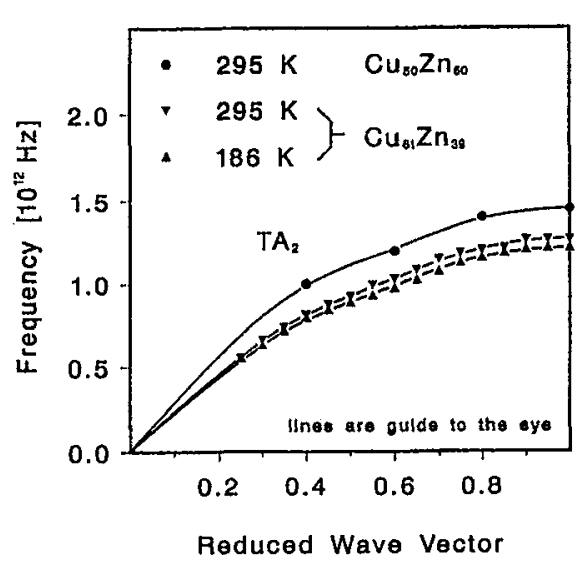

Fig. 7: Phonon branch $\mathrm{TA}_{2}$ of the $\mathrm{SM}$ alloy $\mathrm{Cu}-\mathrm{Zn}$ at $295 \mathrm{~K}$ and at the transition temperature $\mathrm{M}_{\mathbf{s}}$, compared with stoichiometric $\mathrm{CuZn}$.

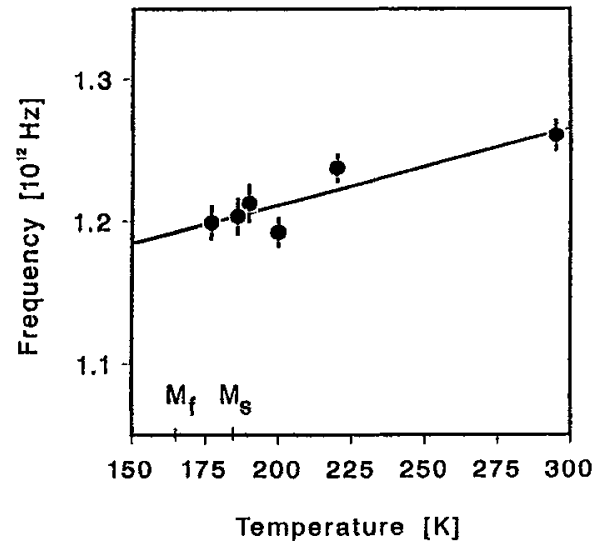

Fig. 8: Phonon frequency of branch $\mathrm{TA}_{2}$ in [110] direction as function of temperature. The values at the zone boundary are shown, representing the frequency change of the whole branch.

below the stoichiometric values. Thus for the $\mathrm{TA}_{2}$ mode, we have very small restoring forces and large dynamic atomic displacements. The behavior of this mode was further investigated as a function of temperature. The result is displayed in Fig. 8: with decreasing temperature, the frequencies decrease only weakly to about $95 \%$ of their room temperature values; the frequency changes and the measured halfwidths of the phonon peaks show no significant wave vector dependence, and there is neither a pronounced 'dip' nor a broadening near $q \approx 2 / 3 q_{\max }$, the wave vector of a soft phonon mode leading to the atomic positions of the (enlarged) martensitic unit cell. The temperature dependence has been followed down to $180 \mathrm{~K}$, i.e. below $\mathrm{M}_{\mathrm{S}}$, where part of the crystal already is transformed into the monoclinic phase.

Experiments in $\mathrm{Cu}-\mathrm{Zn}-\mathrm{Al} / 7 /$ showed a slightly more pronounced dip near $2 / 3 \mathrm{q} \max$, but the overall temperature dependence is very similar.

\subsection{Discussion}

Results of elastic diffuse and inelastic neutron scattering experiments in the austenitic phase of $\beta-\mathrm{Cu}-\mathrm{Zn}$ show the existence of large static and dynamic atomic displacements. The crystal has very weak restoring forces along the directions $\langle 110\rangle$. The weakness can, between limits, be tuned by varying the concentration: reducing the $\mathrm{Zn}$ content results in a specific frequency decrease of the [110] $\mathrm{TA}_{2}$ mode. In a simple picture, on might say that the number of electrons, through electron-phonon interactions, governs the dynamics of this mode.

The temperature dependent softening of the low frequency mode does not show a pronounced anomaly, nor is there a very strong elastic scattering near a critical wave vector. This is in strong contrast to the related system $\beta-\mathrm{Ni}_{62.5} \mathrm{Al}_{37.5} / 8 /$, where a strongly temperature dependent and well pronounced dip in the dispersion curve and temperature dependent elastic scattering around the critical wave vector $\left(\approx 1 / 7 \mathrm{q}_{\max }\right)$ is observed.

To conclude, even in the small family of $\beta$-phase (B2) binary alloys, very different precursor effects of the martensitic phase transition are observed; thus care should be taken in attempting to apply common rules since each system behaves in a unique manner. 


\section{Acknowledgement}

The authors are grateful to Drs. R. Gotthardt, EPF Lausanne, J. van Humbeeck, University of Leuven, and B. Schönfeld, ETH Zürich, for providing samples and helpful discussions. The underlying work was in part supported by the Swiss National Science Foundation.

\section{References}

/1/ G. Kostorz and S. Lovesey, in Treatise on Materials Science and Technology, ed. G. Kostorz, Academic Press (1979); and further chapters therein

/2/ G.L. Squires, Introduction to the Theory of Neutron Scattering, Cambridge University Press (1978)

13/ S.W. Lovesey, Theory of Neutron Scattering from Condensed Matter, Clarendon Press Oxford (1984)

14/ M. Zolliker et al., to be published

/5/ B. Borie, C.J. Sparks, Acta Cryst. A27 (1971) 198

/6/ G. Gilat and G. Dolling, Phys. Rev. 138 (1965) A 1053

$7 /$ G. Guenin, S. Hautecler, R. Pynn, P.F. Gobin, and L. Delaey Scripta Met. 13, 429 (1979)

/8/ S.M. Shapiro, B.X. Yang, G. Shirane, Y. Noda; and L.E. Tanner Phys. Rev. Lett. 62, 1298 (1989) 